\title{
Exact results on superconductivity due to interband coupling
}

\author{
Y. Morita* \\ Institute for Solid State Physics, University of Tokyo, 7-22-1 Roppongi Minato-ku, Tokyo 106, Japan \\ Y. Hatsugai ${ }^{\dagger}$ \\ Department of Applied Physics, University of Tokyo, 7-3-1 Hongo, Bunkyo-ku, Tokyo 113, Japan \\ K. Kohmoto \\ Institute for Solid State Physics, University of Tokyo, 7-22-1 Roppongi Minato-ku, Tokyo 106, Japan
}

(Received 18 July 1995; revised manuscript received 8 December 1995)

\begin{abstract}
We present a family of exactly solvable models at arbitrary filling in any dimensions which exhibit superconductivity with interband pairing. By the use of the hidden SU(2) algebra the Hamiltonians were diagonalized explicitly. The zero-temperature phase diagrams and the thermodynamic properties are discussed. Several properties are revealed which are different from those of the BCS-type superconductor.
\end{abstract}

Superconductivity is one of the most remarkable phenomena in condensed-matter physics. Recently possibilities of a superconductor are proposed by Kohmoto and Takada. ${ }^{1}$ They investigated the superconducting instability of insulators by the mean-field treatment. A two-band system which is insulating without interactions becomes superconducting by a sufficiently large interband attraction. It has many properties which are different from those of the BCS-type superconductors. $^{2}$ Note that the Cooper instability is irrelevant here, since there is no Fermi surface. In Ref.3, possible realization in organic materials is discussed, which is an extension of the Little's idea for the room-temperature superconductor. ${ }^{5}$.

We have constructed a family of exactly solvable models at arbitrary filling in any dimensions which includes the models proposed in Refs. 1 and 3. We have obtained the ground state and the thermodynamic quantities explicitly. Several properties have been revealed. An instability without a Fermi surface, which was proposed by Kohmoto and Takada, is realized in the models. This instability is quite different from the Cooper instability. A finite strength of attraction is needed to produce the superconductivity in contrast to the BCS-type superconductivity. We also note that there are possibilities that our picture realizes in a realistic and complicated model. ${ }^{4}$

Let us consider a two-band model described by the Hamiltonian

$$
\begin{gathered}
\mathscr{H}=\mathscr{H}_{\mathrm{kin}}+\mathscr{H}_{\mathrm{int}}, \\
\mathscr{H}_{\mathrm{kin}}=\sum_{k} \epsilon^{(v)}(k) c_{k}^{(v)^{\dagger}} c_{k}^{(v)}+\sum_{k} \epsilon^{(c)}(k) c_{k}^{(c)^{\dagger}} c_{k}^{(c)}, \\
\mathscr{H}_{\text {int }}=-\frac{U}{N}\left(\sum_{k} c_{k}^{(c)^{\dagger}} c_{-k}^{(v)^{\dagger}}\right)\left(\sum_{k} c_{-k}^{(v)} c_{k}^{(c)}\right),
\end{gathered}
$$

where $c_{k}^{(v)}$ and $c_{k}^{(c)}$ are the fermion annihilation operators for the valence band and the conduction band and $\epsilon^{(v)}(k)$ and $\epsilon^{(c)}(k)$ are the energy dispersions of the valence band and the conduction band, respectively. The momentum vector $k$ takes values in the $d$-dimensional Brillouin zone. We impose a constraint "symmetric condition" on the band structure

$$
\boldsymbol{\epsilon}^{(v)}(k)+\boldsymbol{\epsilon}^{(c)}(-k)=C,
$$

where $C$ is independent of $k$. Without loss of generality we set $C=0$. We set $U$ positive and $\sim O\left(N^{0}\right)$, where $N$ is the number of the momentum points in the Brillouin zone. The interaction is an interband attraction. The spin degrees of freedom are neglected for simplicity, since we do not consider the spin-related quantities here.

Let us sketch the process of the diagonalization. The diagonalization consists of two steps. At first we show the "decoupling property" of the Hamiltonian. Next we map the system to an exactly solvable quantum spin system. Then we can construct all the eigenvalues and the eigenvectors.

We represent the states in the Hilbert space diagrammatically ( see Fig.1). Let us span the Hilbert space by the base vectors

$$
\begin{aligned}
& c_{-p_{1}}^{(v)^{\dagger}} \cdots c_{-p_{N_{e}}^{v}}^{(v)^{\dagger}} c_{q_{1}}^{(c)^{\dagger}} \cdots c_{q_{N_{e}}^{c}}^{(c)^{\dagger}}|0\rangle_{\mathscr{S}} \otimes\left(c_{-k_{1}}^{(v)^{\dagger}} c_{k_{1}}^{(c)^{\dagger}}\right)\left(c_{-k_{2}}^{(v)^{\dagger}} c_{k_{2}}^{(c)^{\dagger}}\right) \\
& \quad \times\left(c_{-k_{3}}^{(v)^{\dagger}} c_{k_{3}}^{(c)^{\dagger}}\right) \cdots\left(c_{-k_{M}}^{(v)^{\dagger}} c_{k_{M}}^{(c)^{\dagger}}\right)|0\rangle_{\mathscr{D}},
\end{aligned}
$$

where $\left\{p_{1}, \cdots, p_{N_{e}^{v}}, q_{1}, \cdots, q_{N_{e}^{c}}\right\}=\mathscr{S}$ and $k_{1}, k_{2}, k_{3}, \cdots, k_{M}$ $\in \mathscr{D}$ (The sets $\mathscr{S}$ and $\mathscr{D}$ will be defined below). Here $|0\rangle_{\mathscr{S}}$ is defined by $c_{k}^{(c)}|0\rangle_{\mathscr{S}}=0(k \in \mathscr{S})$ and $c_{k}^{(v)}|0\rangle_{\mathscr{S}}=0(-k \in \mathscr{S})$. $|0\rangle_{\mathscr{D}}$ is defined by $c_{k}^{(c)}|0\rangle_{\mathscr{D}}=0(k \in \mathscr{D})$ and $c_{k}^{(v)}|0\rangle_{\mathscr{D}}=0(-k \in \mathscr{D})$. Consider a pair which consists of the momentum point $-k$ in the valence band and the momentum point $k$ in the conduction band. We denote the pair by $k$, where $k$ takes values in the Brillouin zone. Define the sets $\mathscr{S}$ and $\mathscr{D}$ as follows. If $k$ is single occupied, $k$ belongs to $\mathscr{S}$. And, if $k$ is empty or double occupied, $k$ belongs to $\mathscr{D}$. Note $\mathscr{S} \cap \mathscr{D}=\phi$ and $\mathscr{A} \cup \mathscr{D}=$ the Brillouin zone.

Let us introduce an operator $\mathscr{P}^{j}$ which is a projection operator to the Hilbert space where $\mathscr{S}$ and $\mathscr{D}$ are fixed to be 


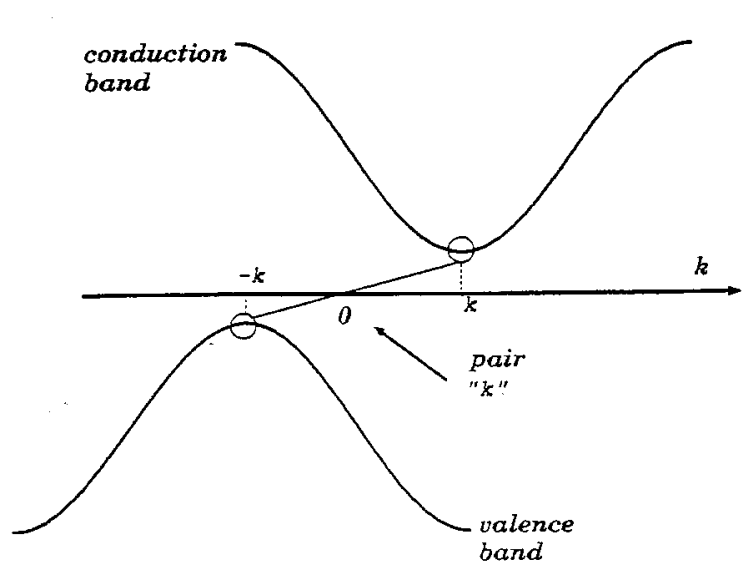

the classification of the pairs

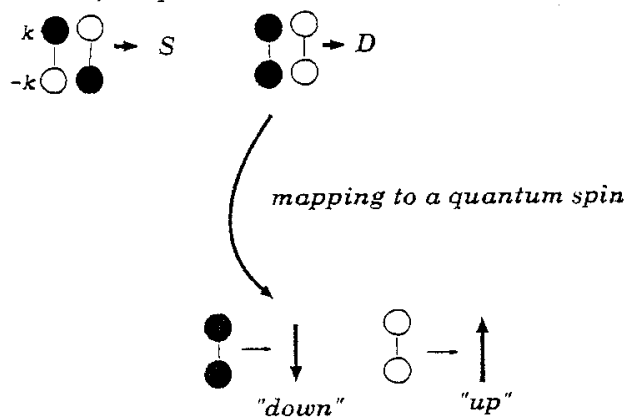

FIG. 1. The classification of the pairs and mapping to a quantum spin.

$\mathscr{S}_{j}$ and $\mathscr{D}_{j}$. The index $j$ denotes how $\mathscr{S}$ and $\mathscr{D}$ are fixed. Using the properties of $\mathscr{P}^{j}$, rewrite the Hamiltonian as

$$
\mathscr{H}=\left(\sum_{j} \mathscr{P}^{j}\right) \mathscr{H}\left(\sum_{j} \mathscr{P}^{j}\right)=\sum_{j} \mathscr{P}^{j} \mathscr{H} \mathscr{P}^{j} .
$$

Using the relation (4), we have

$$
\begin{aligned}
\mathscr{P}^{j} \mathscr{H} \mathscr{P}^{j}= & \mathscr{P}^{j} \mathscr{H}_{\mathrm{kin}} \mathscr{P}^{j}+\mathscr{P}^{j} \mathscr{H}_{\mathrm{int}} \mathscr{P}^{j}=\mathscr{P}^{j}\left(\mathscr{H}_{\mathbf{l}} \otimes \mathbf{1}\right) \mathscr{P}^{j} \\
& +\mathscr{P}^{j}\left(\mathbf{1} \otimes \mathscr{H}_{\mathrm{II}}\right) \mathscr{P}^{j},
\end{aligned}
$$

where $\mathbf{1}$ is an identity operator and $\mathscr{H}_{\mathrm{I}}$ and $\mathscr{H}_{\mathrm{II}}$ are

$$
\begin{gathered}
\mathscr{H}_{\mathrm{I}}=\sum_{k \in \mathscr{S}_{j}} \epsilon^{(v)}(-k) c_{-k}^{(v)^{\dagger}} c_{-k}^{(v)}+\sum_{k \in \mathscr{S}_{j}} \epsilon^{(c)}(k) c_{k}^{(c)^{\dagger}} c_{k}^{(c)}, \\
\mathscr{H}_{\mathrm{II}}=-\frac{U}{N}\left(\sum_{k \in \mathscr{D}_{j}} c_{k}^{(c)^{\dagger}} c_{-k}^{(v)^{\dagger}}\right)\left(\sum_{k \in \mathscr{D}_{j}} c_{-k}^{(v)} c_{k}^{(c)}\right) .
\end{gathered}
$$

Here, the kinetic term and the interaction term decouple, the "decoupling property" of the Hamiltonian.

Now we map the system to an exactly solvable quantum spin system (see Fig. 1). Here the $S U(2)$ algebra hidden in spinless fermions in a two-band system plays a crucial role. ${ }^{6-8}$ Let us define the "spin" operators $\hat{S}_{k}^{+}=\hat{S}_{k}^{x}+i \hat{S}_{k}^{y}$, $\hat{S}_{k}^{-}=\hat{S}_{k}^{x}-i \hat{S}_{k}^{y}$ and $\hat{S}_{k}^{z}$ by $\mathscr{P}^{j} c_{-k}^{(v)} c_{k}^{(c)} \mathscr{P}^{j}, \mathscr{P}^{j} c_{k}^{(c)^{\dagger}} c_{-k}^{(v)^{\dagger}} \mathscr{P}^{j}$ and $\mathscr{P}^{j}\left(\frac{1}{2}-c_{k}^{(c)^{\dagger}} c_{-k}^{(v)^{\dagger}} c_{-k}^{(v)} c_{k}^{(c)}\right) \mathscr{P}^{j}$, respectively, the "total spin" operators $\hat{S}^{\alpha}$ by $\sum_{k \in \mathscr{D}} \hat{S}_{k}^{\alpha}(\alpha=x, y, z)$ and $(\hat{\mathbf{S}})^{2}$ by $\left(\hat{S}^{x}\right)^{2}+\left(\hat{S}^{y}\right)^{2}+\left(\hat{S}^{z}\right)^{2}$. Then we have

$$
\mathscr{P}^{j}\left(\mathbf{1} \otimes \mathscr{H}_{\mathrm{II}}\right) \mathscr{P}^{j}=\mathscr{P}^{j}\left(\mathbf{1} \otimes \mathscr{H}_{\text {spin }}\right) \mathscr{P}^{j},
$$

where $\mathscr{H}_{\text {spin }}$ is defined by

$$
\begin{aligned}
\mathscr{H}_{\text {spin }} & =-\frac{U}{N}\left(\sum_{k \in \mathscr{D}_{j}} \hat{S}_{k}^{x}-i \hat{S}_{k}^{y}\right)\left(\sum_{k \in \mathscr{D}_{j}} \hat{S}_{k}^{x}+i \hat{S}_{k}^{y}\right) \\
& =-\frac{U}{N}\left(\hat{S}^{x}-i \hat{S}^{y}\right)\left(\hat{S}^{x}+i \hat{S}^{y}\right) \\
& =-\frac{U}{N}\left\{(\hat{\mathbf{S}})^{2}-\left(\hat{S}^{z}\right)^{2}-\left(\hat{S}^{z}\right)\right\} .
\end{aligned}
$$

The operators defined above satisfy the relations

$$
\begin{gathered}
{\left[S_{k}^{l}, S_{\tilde{k}}^{m}\right]=i \epsilon_{l m n} S_{k}^{n} \delta_{k \tilde{k}},} \\
\left(\hat{S}_{k}^{x}\right)^{2}+\left(\hat{S}_{k}^{y}\right)^{2}+\left(\hat{S}_{k}^{z}\right)^{2}=\frac{1}{2}\left(\frac{1}{2}+1\right),
\end{gathered}
$$

where $k$ and $\tilde{k}$ take values in $\mathscr{D}_{j}$. Thus $\hat{S}_{k}^{x}, \hat{S}_{k}^{y}$, and $\hat{S}_{k}^{z}$ $\left(k \in \mathscr{D}_{j}\right.$ ) are the components of a $s=\frac{1}{2}$ quantum spin. Now we can identify $k$ with a "site" on which a $s=\frac{1}{2}$ quantum spin is defined. In the language of spin, if the pair $k$ is empty, the spin on the site $k$ is "up" and if the pair $k$ is double occupied, the spin on the site $k$ is "down." Note that, since $k$ takes values in $\mathscr{D}_{j}$, all the pairs we now consider are either empty or double occupied. Now diagonalize $\mathscr{H}_{\text {spin }}$ which can be identified with the Hamiltonian of the quantum spin system $\left(s=\frac{1}{2}\right)$. Define $\left|S, S^{z}\right\rangle$ by an eigenstate of $(\hat{\mathbf{S}})^{2}$ and $\hat{S}^{z}$ which satisfies $(\hat{\mathbf{S}})^{2}\left|S, S^{z}\right\rangle=S(S+1)\left|S, S^{z}\right\rangle$ and $\hat{\mathrm{S} z}|\mathrm{~S}, \mathrm{Sz}\rangle$ $=S z|S, S z\rangle$. The energy is specified by $S$ and $S^{z}$ [see (10)]. There is, however, nontrivial degeneracy which is given by

$$
\frac{\left(2 S_{\max }\right) !(2 S+1)}{\left(S_{\max }-S\right) !\left(S_{\max }+S+1\right) !},
$$

where $N_{\mathscr{D}_{j}}$ is the number of elements in $\mathscr{D}_{j}$ and $S_{\max }$ is $N_{\mathscr{D}} / 2$. This degeneracy is crucial for the thermodynamic properties. Let us consider the state

$$
c_{-p_{1}}^{(v)^{\dagger}} \cdots c_{-p_{N_{e}^{v}}}^{(v)^{\dagger}} c_{q_{1}}^{(c)^{\dagger}} \cdots c_{q_{N_{e}^{c}}}^{(c)^{\dagger}}|0\rangle \otimes\left|S, S^{z}\right\rangle,
$$

where $\left\{p_{1}, \cdots, p_{N_{e}^{v}}, q_{1}, \cdots, q_{N_{e}^{c}}\right\}=\mathscr{S}_{j}$. From the "decoupling property" (7) and the mapping to the quantum spin system (9), it can been seen that this is an eigenvector of $\mathscr{H}$ with an eigenvalue

$$
\begin{aligned}
& \sum_{l=1}^{N_{e}^{v}} \epsilon^{(v)}\left(-p_{l}\right)+\sum_{m=1}^{N_{e}^{c}} \epsilon^{(c)}\left(q_{m}\right)-\frac{U}{N}\left[r^{2}-\left(N_{\mathscr{D}_{j}}+1\right) r\right. \\
& \left.+\frac{N_{e}^{\text {pair }}}{2}\left(N_{\mathscr{D}_{j}}-\frac{N_{e}^{\text {pair }}}{2}+1\right)\right],
\end{aligned}
$$

where $N_{e}^{\text {pair }}$ and $r\left(0 \leqslant r \leqslant N_{\mathscr{D}} / 2, r\right.$ :integer $)$ are defined by $N_{\mathscr{D}_{j}}-2 S^{z}$ and $N_{\mathscr{D}} / 2-S$, respectively. The total number of the fermions is given by $N_{e}^{v}+N_{e}^{c}+N_{e}^{\text {pair }}$. Varying the index $j, \mathscr{H}$ is diagonalized completely.

Now let us consider the physical properties of the system in the thermodynamic limit $(N \rightarrow \infty)$. For simplicity, we consider the half-filled case, namely, $N_{e}^{v}+N_{e}^{c}+N_{e}^{\text {pair }}=N$. When the interaction is absent, the system is insulating. 

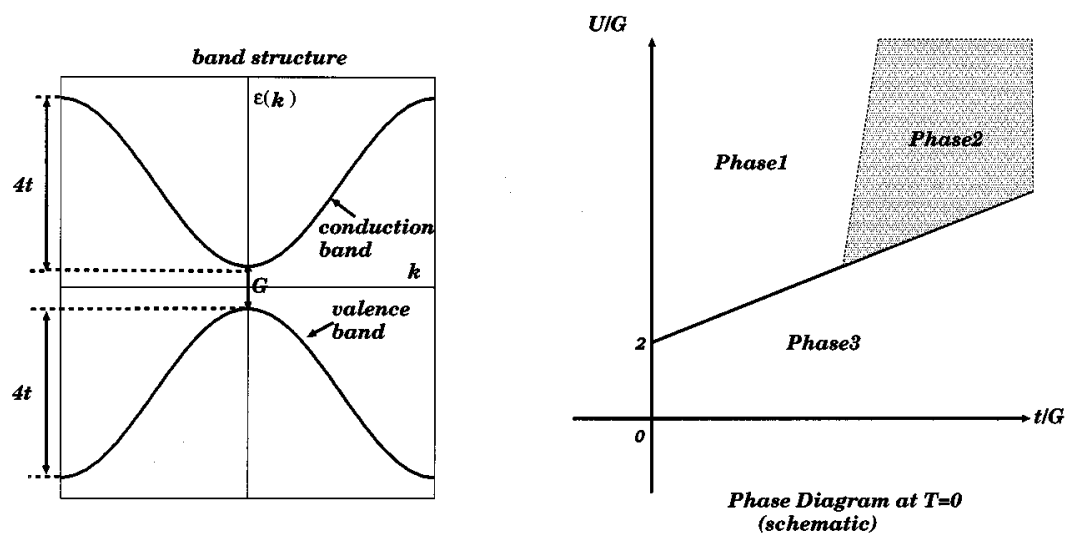

FIG. 2. The one-dimensional two-band model, where $\epsilon^{(c)}(k)=-2 t \cos k+2 t+G / 2$ and $\epsilon^{(v)}(k)=2 t \cos k-2 t-G / 2$. The zero-temperature phase diagram.

Let us first consider the zero-temperature phase diagrams. The ground state was obtained by minimizing the energy (14). The competition between the kinetic term and the interaction term gives a rich phase diagram. We present the phase diagrams of two cases: the one-dimensional two-band model, as shown in Fig. 2, and a system with a constant density of states, which resembles that of the twodimensional systems (Fig. 3). We find three types of different phases as shown in Figs. 2 and 3. All the phases are separated by the first-order phase transitions. The phases are characterized by $\Delta$ (= the amplitude of the off-diagonal long-range order for the $s$-wave superconductivity ${ }^{11}$ ) which is defined by

$$
\Delta=\sqrt{\frac{1}{N^{2}} \int d x \int d y\left\langle\psi^{(c)^{\dagger}}(x) \psi^{(v)^{\dagger}}(x) \psi^{(v)}(y) \psi^{(c)}(y)\right\rangle} .
$$

Here $\quad \psi^{(c)}(x)=1 / \sqrt{N} \Sigma_{k} e^{i k x} c_{k}^{(c)} \quad$ and $\quad \psi^{(v)}(x)$ $=1 / \sqrt{N} \Sigma_{k} e^{i k x} c_{k}^{(v)}$. When $\Delta$ takes finite value, the pairing of electrons occurs as in the BCS superconductivity. The contents of the three phases are as follows:

Phase 1: $\Delta=0.5$, which is the upper bound for $\Delta$. It is superconducting. ${ }^{11,12}$

Phase 2: $0<\Delta<0.5$. It is also superconducting.

Phase 3: $\Delta=0$. The ground state is a band insulator as the noninteracting case.
Note that a sufficiently large attraction is needed to produce the superconductivity, which is totally different from the BCS superconductivity.

Now let us discuss the Meissner effect, namely, estimate the superfluid density $N_{s} . \quad N_{s}$ is defined by $m c / e^{2}|| \mathbf{j}\|/\| \mathbf{A} \|$, where $m$ denotes the effective mass, $\mathbf{j}$ is the current density, and $\mathbf{A}$ is the vector potential. Since we have diagonalized the Hamiltonian explicitly, it is straightforward to obtain $N_{s}$ by the use of the Kubo formula. In the insulating phase we obtain $N_{s} / N=0$ and there is no Meissner effect. This is the direct consequence of the effective mass theorem. ${ }^{13}$ In the superconducting phase we can also obtain $N_{s} / N=1+O(1 / U)$ in the large $U$ limit, which means the Meissner effect.

Next we consider the thermodynamic properties. For simplicity, we consider a system with flat bands $\left(\epsilon^{(v)}=-\epsilon \epsilon^{(c)}=\epsilon\right.$.) When the two bands degenerate, namely $\epsilon=0$, the thermodynamic properties are investigated by Thouless. ${ }^{8}$ The grand partition function is

$\mathscr{Z}_{\text {grand }}=\sum_{\substack{N_{e}^{v}, N_{e}^{c} \\ 0 \leqslant N_{e}^{v}+N_{e}^{c} \leqslant N}} \sum_{\substack{r ; \text { integar } \\ 0 \leqslant r \leqslant N_{\mathscr{D}_{j} / 2}}} \sum_{N_{e}^{\text {pair }}=2 r}^{2 N_{\mathscr{D}_{j}}-2 r} \mathscr{C} \exp (-\beta \mathscr{E})$,

where $\mathscr{C}$ and $\mathscr{E}$ is defined by
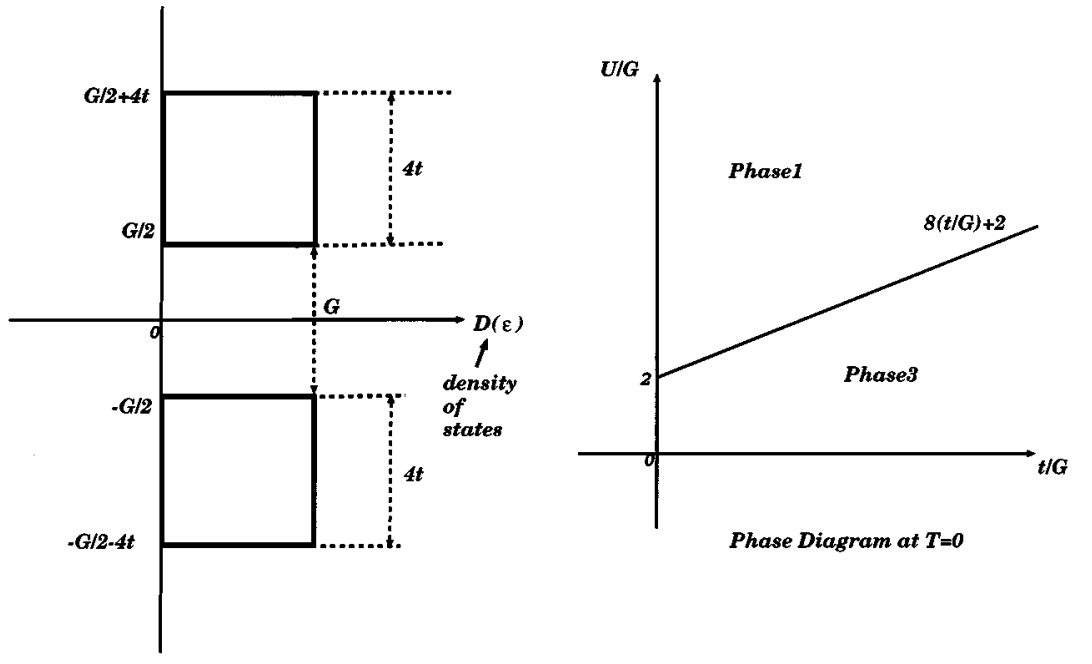

FIG. 3. The model which has a constant density of states. The density of states and the zerotemperature phase diagram. 


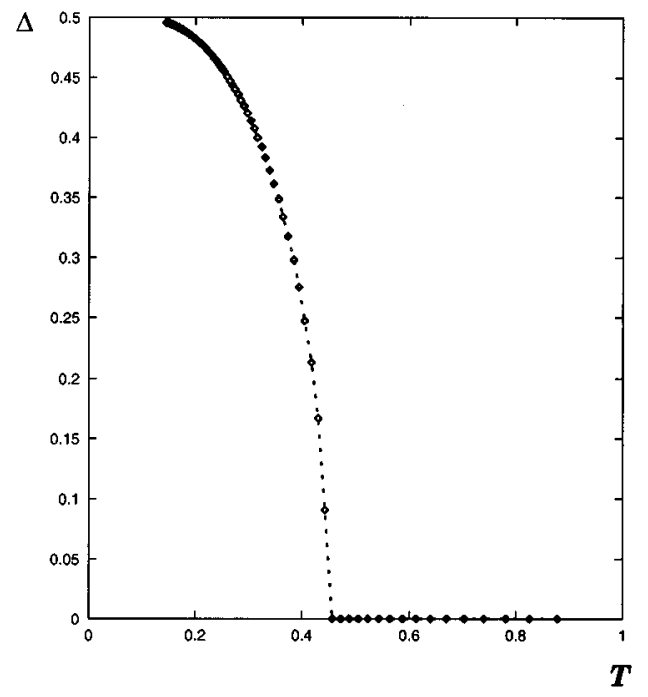

order parameter

$$
\mathscr{C}=\frac{N !}{N_{\mathscr{D}_{j}} ! N_{e}^{v} ! N_{e}^{c} !} \frac{N_{\mathscr{D}_{j}} !\left(N_{\mathscr{D}_{j}}-2 r+1\right)}{r !\left(N_{\mathscr{D}_{j}}-r+1\right) !}
$$

and

$$
\begin{aligned}
\mathscr{E}= & -\mu\left(N_{e}^{v}+N_{e}^{c}+N_{e}^{\mathrm{pair}}\right)-N_{e}^{v} \epsilon+N_{e}^{c} \epsilon-\frac{U}{N}\left\{r^{2}-\left(N_{\mathscr{D}_{j}}+1\right) r\right. \\
& \left.+\frac{N_{e}^{\text {pair }}}{2}\left(N_{\mathscr{D}_{j}}-\frac{N_{e}^{\mathrm{pair}}}{2}+1\right)\right\} .
\end{aligned}
$$

In the thermodynamic limit $(N \rightarrow \infty)$ we use the saddle-point method. The chemical potential is set $\mu=0$ and the system is half-filling. A direct calculation leads to analytic forms of the thermodynamic quantities. For example, $\Delta(T)$ is given by

$$
\Delta(T)=0.5 \frac{y^{2}-1}{y^{2}+a y+1},
$$

where $y$ is the largest root of $\ln x$ $=\frac{1}{2} U T^{-1}(x-1)(x+1)\left(x^{2}+a x+1\right)^{-1}$ and $a=e^{\epsilon / T}+e^{-\epsilon / T}$. As shown in Fig. 4, the second-order phase transition occurs at a finite temperature. The critical temperature $T_{c}$ is proportional to $U$ when $U \gg \epsilon$. The entropy $S(T)$ per unit cell is given by

$$
\begin{aligned}
S(T) / N= & \ln \left(y^{2}+a y+1\right)-\epsilon T^{-1} \frac{\left(e^{\epsilon / T}-e^{-\epsilon / T}\right) y}{y^{2}+a y+1} \\
& -0.5 U T^{-1} \frac{y(2 y+a)\left(y^{2}-1\right)}{\left(y^{2}+a y+1\right)^{2}} .
\end{aligned}
$$

The heat capacity $\left[=T(\partial S / \partial T)_{V}\right]$ per unit cell is shown in Fig. 4. In the superconducting phase it behaves as $A \exp (-B / T)$ at a sufficiently low temperature, where $A$ is a constant and $B=U / 2-2 \epsilon$ is the excitation gap. In the hightemperature phase it is a decreasing function of $T$, since the band widths are finite.

We find that $\Delta(T=0) / T_{c}$ and $\Delta C / C_{n}$ are not universal in contrast to the BCS-type superconductivity, where $\Delta C$ is the
FIG. 4. The temperature dependence of the order parameter and the heat capacity when $\epsilon=0.3$ and $U=2$. jump of the heat capacity at $T=T_{c}$ and $C_{n}$ is the heat capacity at $T=T_{c}+0$. A more detailed study of the thermodynamic properties will be presented elsewhere.

The half-filled case considered here seems to be most prospective to be realized. The crucial point is the origin of the attractive interaction. One of the possible candidates is the exciton mechanism proposed in Refs. 3 and 5. There the attraction is envisaged as arising from a polarizable medium sandwiched between the two chains, where the "effective" interaction between electrons in different chains becomes statically attractive. This is because electrons share positive charge induced in the medium. They have confirmed that there are cases in which this attractive interaction is stronger than the direct Coulomb repulsion between electrons in different chains. ( In Ref. 9, another example of attraction was proposed in the two-band repulsive Hubbard model. The electrons in one band experience attractive interaction mediated by an accompanying Mott-insulator band.) Then, if we consider the filling other than half-filling, the excitonelectron interaction which leads to the attraction is reduced considerably by screening. Thus the half-filling case is best for our purpose. Without the screening, a strong attraction is rather easily achieved. ${ }^{10}$

In summary, a recent proposal by Kohmoto and Takada of the pairing state between a conduction electron and a valence electron was investigated through a family of exactly solvable models. We obtained all the eigenvalues and the eigenvectors explicitly. The zero-temperature phase diagrams were obtained. The superconducting instability without a Fermi surface which was proposed by Kohmoto and Takada were confirmed. It was also proved that a sufficiently large attraction between states in the two bands is needed to produce superconductivity. The thermodynamic properties were also dicussed. The properties are quite different from those of the BCS-type superconductor. The models we consider may be realized in specially synthesized double-chain organic materials. Although we have presented the results for the cases where fully analytical treatments are possible, the results for the more general cases are not different from the present cases in essential ways. They will be presented elsewhere. 
*Electronic address: morita@kodama.issp.u-tokyo.ac.jp

†Electronic address: hatsugai@tansei.cc.u-tokyo.ac.jp

${ }^{1}$ M. Kohmoto and Y. Takada, J. Phys. Soc. Jpn. 59, 1541 (1990).

${ }^{2}$ J. Bardeen, L. N. Cooper, and J. R. Schrieffer, Phys. Rev. 108, 1175 (1957).

${ }^{3}$ Y. Takada and M. Kohmoto, Phys. Rev. B 41, 8872 (1990).

${ }^{4}$ K. Kuroki, H. Aoki, and Y. Takada, J. Phys. Soc. Jpn. 61, 1161 (1992).

${ }^{5}$ W. A. Little, Phys. Rev. 134, A1416 (1964).

${ }^{6}$ P. W. Anderson, Phys. Rev. 112, 1900 (1958).
${ }^{7}$ Y. Wada, F. Takano, and N. Fukuda, Prog. Theor. Phys. 19, 597 (1958).

${ }^{8}$ D. J. Thouless, Phys. Rev. 117, 1256 (1960).

${ }^{9}$ H. Aoki and K. Kuroki, Phys. Rev. B 42, 2125 (1990).

${ }^{10}$ D. Davis, H. Gutfreund, and W. A. Little, Phys. Rev. B 13, 4766 (1976).

${ }^{11}$ C. N. Yang, Rev. Mod. Phys. 34, 694 (1962).

${ }^{12}$ G. Sewell, J. Stat. Phys. 61, 415 (1990).

${ }^{13}$ See, for example, N. W. Ashcroft and N. D. Mermin, Solid State Physics (Saunders College, Philadelphia, PA, 1976), p. 765. 